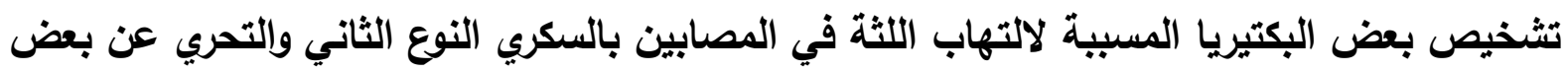

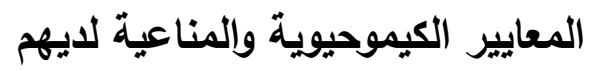

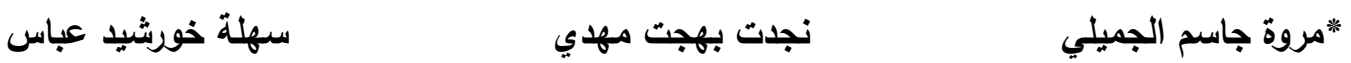 \\ قسم علوم الحياة / كلية العلوم/ جامعة كركوك
}

* E-mail: Marwa.jassim $1987 @$ gmali.com

$$
\text { (أستلم 2018/ } 10 \text { / 2018 }
$$

\begin{abstract}
الملخص
هدف البحث الى التحري عن الأنواع البكتيرية المسببة لإلتهاب اللثة، حيث عُزلت البكتيريا الموجبة لصبغة غرام بنسبة

(89\%) أكثر من البكتيريا السالبة لصبغة غرام وبواقع 60 عزلة من بكتبريا Streptococcus viridans و 5 عزلات من بكتيريا Escherichia coli الكيموحيوية ارتقاعاً معنوياً في تركيز الكولسترول الكلي تحت مستوى معنوية (P>0.05) لدى مجموعة التهاب اللثة والسكري، و و

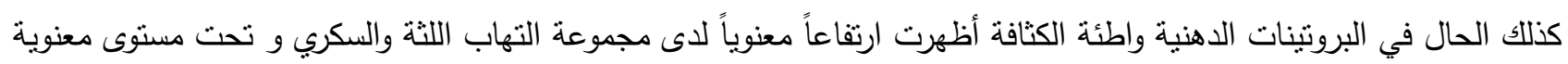

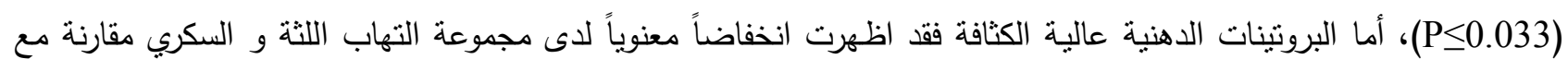

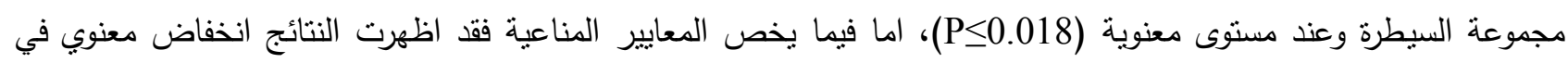

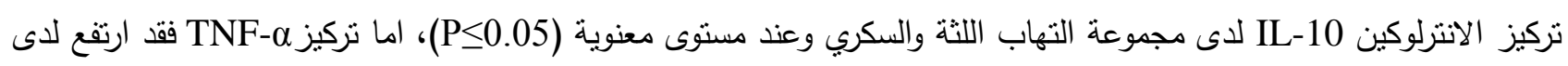
مجموعة التهاب اللثة والسكري وعند مستوى معنوية (P (P)0.05).
\end{abstract}

الكلمات الدالة: التهاب اللثة، السكري، المعايير الكيموحيوية، الحركيات الخلوية، TNF- $\alpha$ ،IL-10.

\section{Diagnosis of some Bacteria Causing Gingivitis in People with Type 2 Diabetes and Investigating some of Their Biochemical and Immunological Parameters}

\author{
Marwa J. Aljumaily \\ Najdat B. Mehdi \\ Sahla KH. Abbas \\ Department of Biology /College of Science / University of Kirkuk
}

\begin{abstract}
The research aimed to isolate and identify the types of bacteria which cause gingivitis. Gram positive bacteria were isolated by $89 \%$ more than gram-negative bacteria, 60 isolates were Streptococcus viridans and 5 isolates were Escherichia coli. The biochemical study showed a significant increase in concentration of total cholesterol $(\mathrm{P} \leq 0.05)$ in the gingivitis and diabetes groups, as well as in very low density lipoproteins showed a significant increase in gingivitis and diabetes group ( $\mathrm{P} \leq 0.033$ ), High-density lipoproteins showed a significant decrease in the group of gingivitis and diabetes compared to the control group at a significant level $(\mathrm{P} \leq 0.018)$. In the immunological parameters, the results showed a significant decrease in the concentration of IL-10 in the group gingivitis and diabetes $(\mathrm{P} \leq 0.05)$. The concentration of TNF- $\alpha$ increased in gingivitis and diabetes at a significant level $(\mathrm{P} \leq 0.05)$.
\end{abstract}




$$
\text { مروة جاسم الجميلي وآخرون }
$$

Keywords: gingivitis, diabetes, biochemical parameters, cytokines , IL-10, TNF- $\alpha$.

\section{المقدمة}

إن أمراض تسوس الأسنان وأمراض اللثة تعد من المشاكل الرئيسية الأكثر أهمية في الصحة العامة، حيث يحتوي التجويف

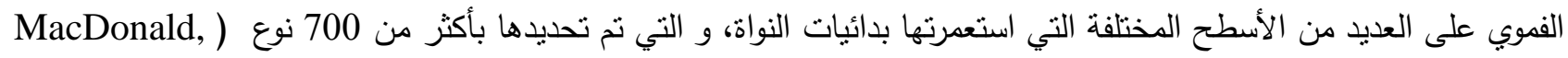
2015)، التهاب اللتة Gingivitis هو مرض التهابي مزمن، يحدث فيه خلل في التوازن بين تفاعلات المضيف والميكروبات و هذا هو المفتاح لبداية ظهور المرض وتطوره (Alvarez et al., 2018). إن من أكثر الأنواع البكتيرية المسبية لإلتهاب اللثة وتتخر الأسنان هي البكتيريا المسبحية .Streptococcus sp (فزاع، 2013)، ومن أكثر هذه الأنواع المسؤولة عن التهاب اللثة هي المسبحيات الفموية (Viridans group Streptococci (VGS) ، فيما ذكر (Al-Abdul and Hussein, 2017) بأن فئريا بكتيريا Staphylococci هي المهيمنة في الإلتهابات اللنثوية. داء السكري هو واحد من أكثر الأمراض المزمنة شيوعا حيث تم تعريفه على أنه مجموعة من أمراض التمثيل الغذائي و

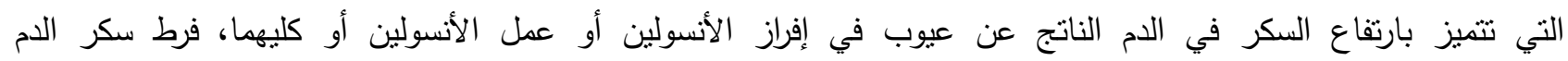
Hyperglycemia

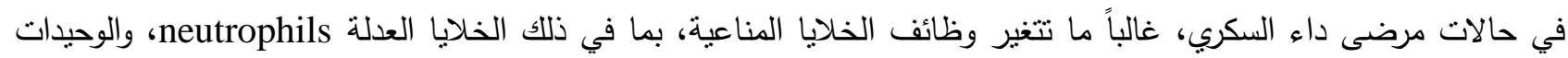

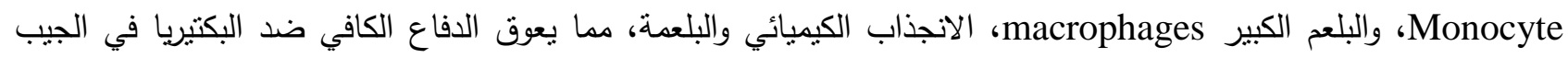
اللثوي وزيادة كبيرة في تدمير الغشاء اللثوي في مرضى السكري، على الرغم من انخفاض في كفاءة وظيفة العدلات، إلا إنه قد يكون هناك استجابة فائقة لخط خلية الوحيدات/ البلعمية عند مواجهة أي مستضدات بكتيريـة و كنتيجة لذلك يتم زيادة إنتاج الحركيات الخلوية البادئة للالتهابات. في مرضى السكري، هناك إنتاج مستويات عالية من عامل نخر الورم (TNF(Kripal,2017)، حيث تؤدي الحركيات الخلوية Cytokines) دوراً مهماً في تحطيم/تصليح الأنسجة (Zhang et al., 2017).

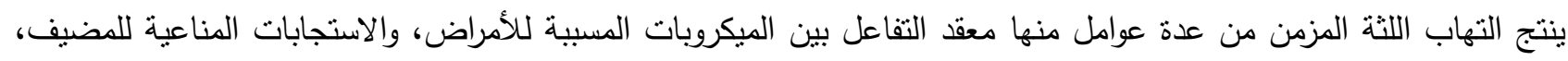

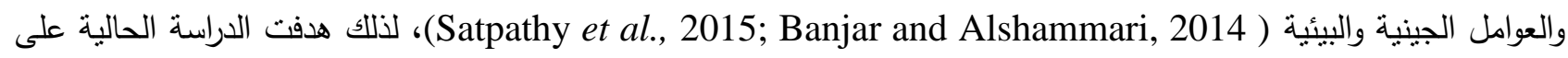
إختبار بعض المعايير الكيموحيوية والمناعية لدى المصابين بالتهاب اللثة فقط Gingivitis ومقارنتها مع المصابين بالتهاب اللثة لاى مرضى السكري النوع الثاني.

\section{المواد وطرائق العمل}

1- جمع العينات و التشخيص المختبري نم جمع 100 مسحة من لثة مرضى مقسمين الى مجموعتين ومن كلا الجنسين، الأولى مجموعة المرضى المصابين بإلتهاب اللثة فقط و الثانية مجموعة المرضى المصابين بإلتهاب اللثة مع السكري. حيث نم جمع العينات من المراجعين لمركز الأسنان

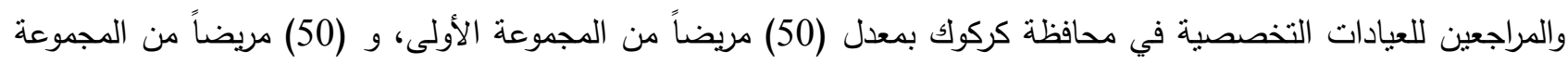

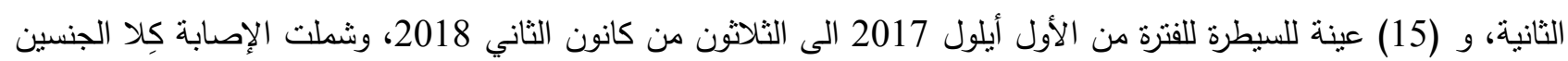

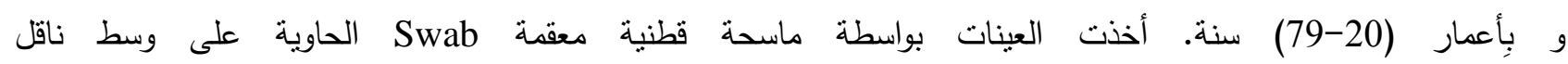

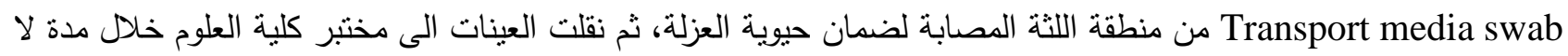
تتجاوز الساعتين لغرض الزرع والتقفية والتشخيص، وإجراء بعض الفحوصات الكيموحيوية في مختبر الصحة العامة في كركوك.

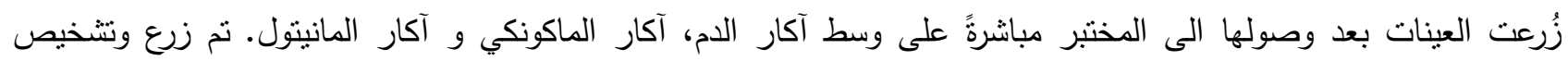


Forbes et al., 2007; Collee et al., 1996) العينات حسب طرائق العمل التقليدية والدذكورة في .( Yadav, 2016 ؛Tille, 2014; 2- المعايير الكيموحيوية و المعايير المناعية تم تقدير تراكيز الكلوكوز في مصل الدم إنزيمياً، وذلك باستخدام العدة الخاصة بشركة Cromatest الإسبانية تم تقدير تركيز الكوليسترول الكلي والكليسريدات الثلاثثة و تركيز البروتينات الدهنية عالية الكثافة للكوليسترول في مصل الدم باستخدام عدة (kit) الخاصة بكل فحص والمجهزة من قِبل شركة (AGAPPE) السويسرية. تم استخدام تقنية الإمتزاز المناعي المرتبط

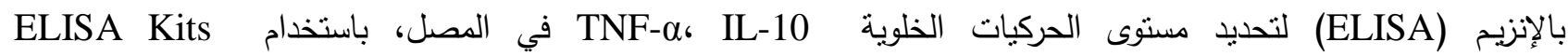
KOMABIOTECH, Germany) 3- 3 التحليل الإحصائي SPSS) Statistical Package for Social أجريت التحليلات الإحصائية باستعمال البرنـامج الإحصائي الجاهـز (Science بالاعتماد على طريقة One-way ANOVA وبمستوى احتمالية P<0.05 لاستخراج الفروقات المعنوية.

\section{النتائج والمناقثنة}

تم تتخيص 108 عزلة بكتيرية من العينات قيد الدراسة حيث عزلت 94 عزلة بكتيرية موجبة لصبغة غرام بواقع 55 عزلة من مجموعة التهاب اللثة والسكري و39 عزلة بكتيرية من التهاب اللثة فقط، تتضمن أجناس .Streptococcus sp. و Staphylococcus sp.

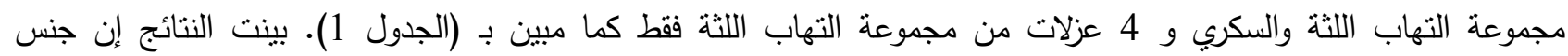
Streptococci وبعض التهابات الفم حيثُ تُسمى بالأنواع المولدة للنخر، تتفق هذه النتائج مع ما توصيّل إليه (فزاع، 2013) و (هادي وآخرون، 2012)، وهذا يتتاقض مع ما توصل اليه كل من (Al-Abdul and Hussein,2017; Jalal et al.,2017) على أن بكتيريا Staphylococcus هي الأكثر شيوعاً في التهاب اللثة، بالإضافة إلى إن جنس Streptococci من الأجناس التي تُهيئ إحداث أمراض إنتهازية Opportunistic infection من قبل المجاميع الأخرى من جنس Streptococci منسيا

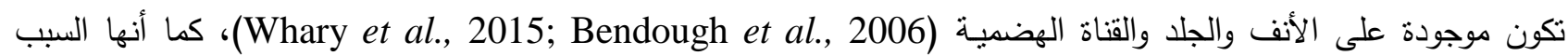
الرئيسي لعدوى المستشفيات الحادة (Hetem et al., 2017)، ويرجع سبب هذا الانتشار في غير موطنها الى امتلاكها آليات مقاومـة سريعة، سهولـة الانتشار والمتمثلة بالبلازميدات من خلال عملية الإقتران والتحول، بالإضافة إلى امتلاكها مستضدات

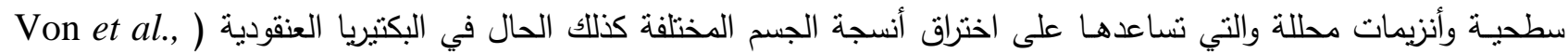
2016). أما البكتيريا السالبة لصبغة غرام كانت أقل نسبةً مقارنة مع البكتيريا الموجبة لصبغة غرام وحسب ما أكدته دراسات عديدة بأن معظم البكتيريا السالبة لصبغة غرام يكون مصدرها التهاب الجهاز التتفسي أو القناة المعديـة المعويـة وهذا يتفق مع كل من (فزاع، 2013؛ بuffnagle et al., 2017 بsus). 
الجدول 1: الأنواع البكتيرية المعزولة من التهاب اللثة

\begin{tabular}{|c|c|c|c|}
\hline 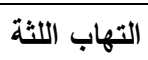 & التهاب اللثة +السكري & الأنواع البكتيرية المعزولة & 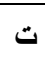 \\
\hline \multicolumn{2}{|c|}{ عدد العزلات } & \multicolumn{2}{|l|}{ البكتبريا الموجبة لصبغة غرام } \\
\hline 15 & 20 & Streptococcus mutans & 1 \\
\hline 3 & 5 & Streptococcus salivarius & 2 \\
\hline 7 & 10 & Streptococcus anginosus & 3 \\
\hline 2 & 4 & Streptococcus pyogenes & 4 \\
\hline 3 & 5 & Enterococcus faecalis & 5 \\
\hline 5 & 7 & Staphylococcus aureus & 6 \\
\hline 4 & 4 & Staphylococcus epidermidis & 7 \\
\hline \multicolumn{2}{|c|}{ عدد العزلات } & \multicolumn{2}{|l|}{ البكتبريا السالبة لصبغة غرام } \\
\hline 2 & 4 & Proteus merabilis & 1 \\
\hline 1 & 4 & Escherichia coli & 2 \\
\hline 1 & 2 & Pseudomonas aeruginosa & 3 \\
\hline 43 & 65 & \multicolumn{2}{|l|}{ المجموع } \\
\hline
\end{tabular}

أما بالنسبة لنتائج الفحوصات الكيموحيوية نسبة الكلوكوز في الدم فقد أظهرت ارتفاعا معنويا في مستوى معدل الكلوكوز،

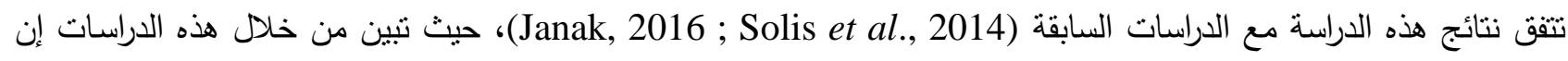

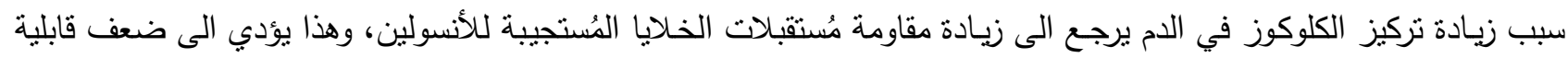
الأنسولين في حمل الكلوكوز الى داخل الخلايا مما يؤدي الى تراكم الكلوكوز في الدم. كما كان هنالك فروق معنوية في مسنوى

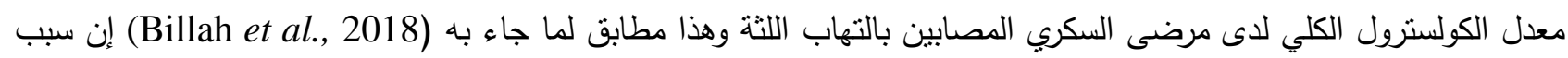

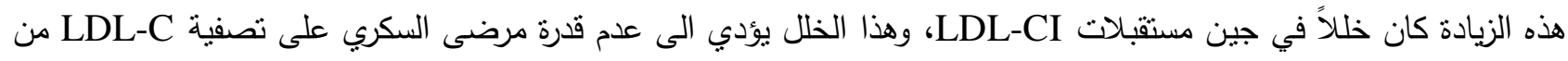

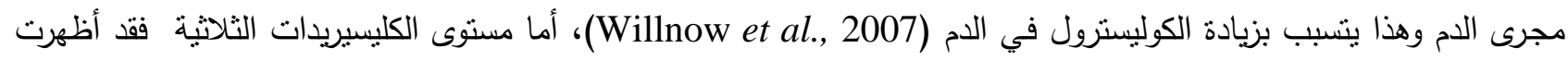

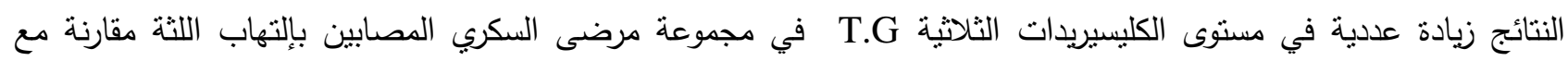
مجموعة المرضى المصابين بإلتهاب اللثة فقط و مجموعة السيطرة (Khursheed et al.,2011) حين لاحظوا إرتفاع نسبة تركيز

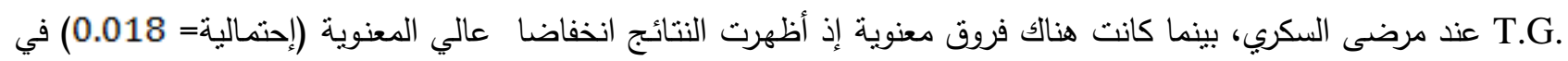

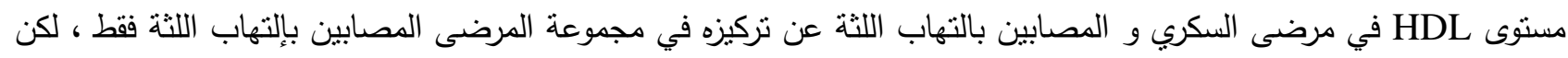

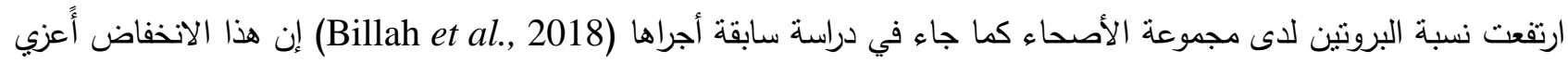
إلى تغيرات في وظائف الكبد، مما سبب تثتيط في إنتاج

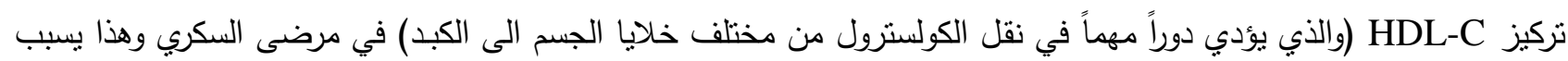
خطراً للإصابة بتصلب الثرايين. والبروتينات الدهنية واطئة الكثافة لم تسجل فروقاً معنوية بحسب النتائج المذكورة. إن التغيرات

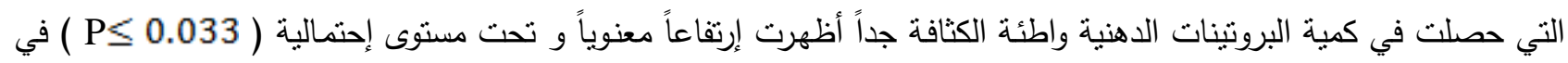

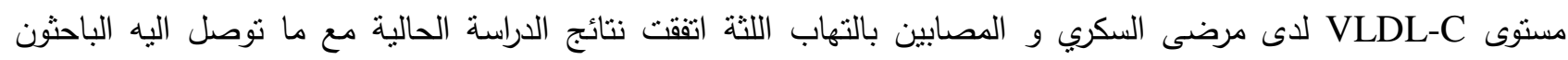

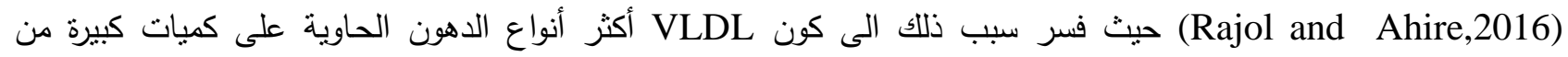
الكليسريدات الثلاثية، يقوم انزيم اللايبوبروتين لايبيز (LPL) المتواجد في العضلات وبطانة أوعية الأنسجة الدهنية بتحليل الكليسريدات الثلاثنة في VLDL، وتكون نواتج هذا التحلل أحماض دهنية و التي تؤخذ من قبل الأنسجة الجسمية، ويتم تأكسدها هناك لغرض الحصول على الطاقة، أو تخزن بشكل الكليسريدات الثلاثية (Mittendorfer, 2005). 
الجدول 2: المعاييز الكيموحيوية لاى المجاميع المدروسة

\begin{tabular}{|c|c|c|c|c|c|c|}
\hline \multicolumn{6}{|c|}{ المعـــل + الخطأ القياسـي } & \multirow{2}{*}{$\begin{array}{ll}\overline{3} & \overline{3} \\
3 & 3 \\
3 & 3\end{array}$} \\
\hline VLDL $\mathrm{mg} / \mathrm{dl}$ & LDL mg/dl & HDL mg/dl & T.G. mg/dl & T.C mg/dl & GLUCOSE mg/dl & \\
\hline $0.352 \pm 0.688^{\mathrm{b}}$ & $0.533 \pm 1.513^{\mathrm{a}}$ & $0.1598 \pm 0.9375^{b}$ & $0.823 \pm 1.550^{\mathrm{a}}$ & $0.719 \pm 3.22^{\mathrm{b}}$ & $17.08 \pm 103.47_{\mathrm{b}}$ & التهاب اللثة \\
\hline $0.586 \pm 1.050^{\mathrm{a}}$ & $1.620 \pm 2.300^{\mathrm{a}}$ & $0.1982 \pm 0.7750^{c}$ & $2.253 \pm 2.362^{\mathrm{a}}$ & $3.79 \pm 5.35^{\mathrm{a}}$ & $104.2 \pm 216.9^{\mathrm{a}}$ & التهاب اللثة + \\
\hline $0.1996 \pm 0.6625^{\mathrm{b}}$ & $0.912 \pm 1.950^{\mathrm{a}}$ & $0.2357 \pm 1.0875^{\mathrm{a}}$ & $0.450 \pm 1.263^{\mathrm{a}}$ & $0.761 \pm 3.213^{\mathrm{b}}$ & $9.95 \pm 101.25^{\mathrm{b}}$ & 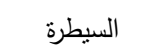 \\
\hline
\end{tabular}

* تُشير الأحرف المتثابهـة الى عدم وجود فروقــات معنوية

انخفض معدل الأنترليوكين-10 لدى مرضى السكري المصابين بالتهاب اللثة بينما ارتفع معدل مستوى مقارنة مع مجموعة التهاب اللثة فقط والسيطرة جاءت هذه النتائج مطابقة لما توصل اليه (Norppa, 2012). ارتبط مرض التهاب التهاب

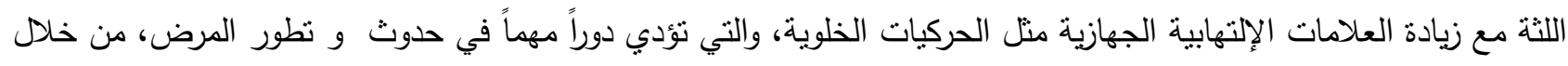

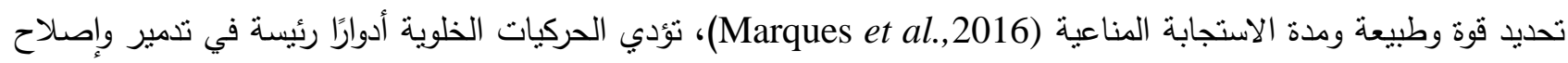
الأنسجة (Zhang et al., 2017)، IL-10 أعثبر من الحركيات الخلوية المضادة للإلتهاب لقدرتها على تثبيط إنتاج الحركيات الخلوية البادئة للإلتهاب منل (IL-6 و IL-8 و ILNF- و IL-1 (Grover et al., 2016)، الحركي الخلوي-10 من الحركيات

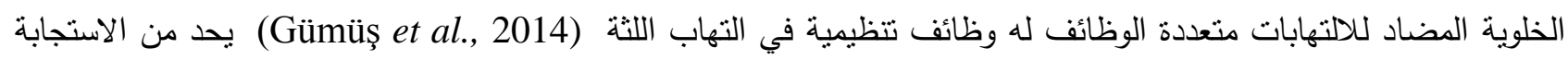
المناعية المفرطة، تدمير الأنسجة وارتتاف العظام لدى المصابين بالتهاب اللثة، عن طريق تتبيط إنتاج الحركيات الخلوية البادئة

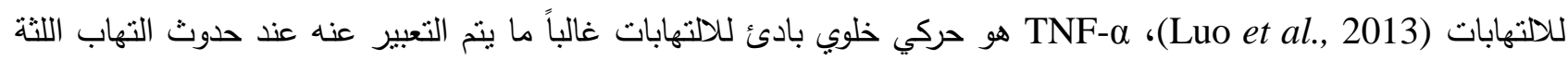

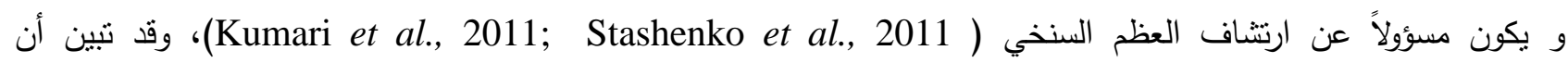

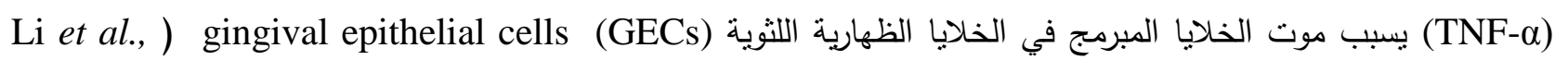

الجدول 3: المعايير المناعية لاى المجاميع المدروسة

\begin{tabular}{|c|c|c|}
\hline \multicolumn{2}{|c|}{ 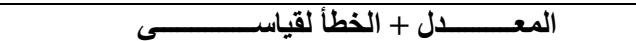 } & \multirow[t]{2}{*}{ المجاميـــع المدروسة } \\
\hline TNF- $\alpha \mathrm{pg} / \mathrm{ml}$ & IL-10 pg/ml & \\
\hline $6.03 \pm 63.76^{\mathrm{b}}$ & $9.1 \pm 141^{\mathrm{a}}$ & 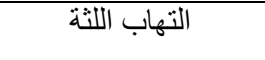 \\
\hline $8.70 \pm 62.21^{\mathrm{a}}$ & $11122.11^{\mathrm{b}} \pm$ & التهاب اللثة + السكري \\
\hline $2.31 \pm 45.63^{\mathrm{c}}$ & $9.91 \pm 144^{\mathrm{a}}$ & السيطرة - م السئ \\
\hline
\end{tabular}

• • تُشير الأحرف المنتشابهة الى عدم وجود فروقات معنوية

\section{المصادر العربية}

فزاع، سعاد عبد (2013). تأثثر المستخلص المائي لإزهار القرنفل على بعض العزلات البكتيرية المسببة لالتهاب اللثة. مجلة

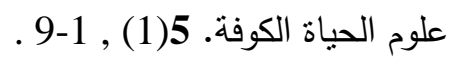
هادي، عدي متعب، مهان، عدنان؛ رزاق، زهير صادق (2012). دراسة بكتريولوجية لعزل وتتخيص البكتريا المسببة لتسوس

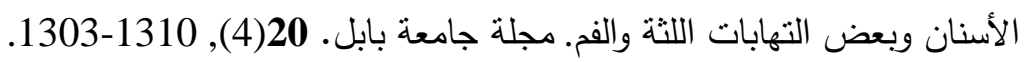




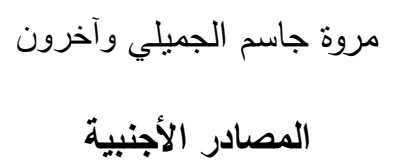

Al-Abdul, A.A.; Hussein, I.K. (2017). Isolation and identification of bacteria from diabetic and nondiabetic patients with periodontitis. Donnish J. Microbiol. and Biotechnol. Research. 4(2), 004-009.

Alvarez, C.; Rojas, C.; Rojas, L.; Cafferata, E.A.; Monasterio, G.; Vernal, R. (2018). Regulatory T lymphocytes in periodontitis: a translational view. Mediators of Inflammation. 2018, 7806912, (10).

Banjar, W.; Alshammari M.H. (2014). Genetic factors in pathogenesis of chronic periodontitis. $J$. Taibah University for Sci., 9, 245-247.

Bendouah, Z.; Barbeau, J.; Hamad, W.A.; Desrosiers, M. (2006). Biofilm formation by Staphylococcus aureus and Pseudomonas aeruginosa is associated with an unfavorable evolution after surgery for chronic sinusitis and nasal polyposis. Otolaryngology Head and Neck Surgery, 134(6), 991-996.

Billah, M.M.; Rana, S.M.; Akter, N.; Hossain, M.S. (2018). Analysis of serum electrolyte and lipid profile in young Bangladeshi female with Type II Diabetes. Cogent Biol., 4(1), 1431474.

Cicmil, S.; Mladenović, I.; Krunić, J.; Ivanović, D.; Stojanović, N. (2018). Oral alterations in diabetes mellitus. Balkan J. Dental Medicine. 22(1), 7-14.

Collee, J.G.; Fraser, A.G.; Marmion, B.P.; Simmons, A. (1996). "Mackie McCartney Practical Medical Microbiology". $14^{\text {th }}$ ed., Longman Singapore Publishers Ltd., Singapore.

Forbes, B.A.; Sahm, D.F.; Weissfeld, A.S. (2007). "Bailey and Scott's Diagnostic Microbiology". $12^{\text {th }}$ ed. Mosby. Inc. St. Louis. USA. pp.166-167.

Grover, H.S.; Kapoor, S.; Singh, A. (2016). Effect of topical simvastatin (1.2 mg) on gingival crevicular fluid interleukin-6, interleukin-8 and interleukin-10 levels in chronic periodontitis-A clinicobiochemical study. J. Oral Biology and Craniofacial Research. 6(2), 85-92.

Gümüş, P.; Nizam, N.; Lappin, D.F.; Buduneli, N. (2014). Saliva and serum levels of B-cell activating factors and tumor necrosis factor- $\alpha$ in patients with periodontitis. $J$. Periodontol., 85(2), 270-280.

Hetem, D.J.; Rooijakkers, S.H.; Ekkelenkamp, M.B. (2017). "Staphylococci and Micrococcus in Infectious Diseases". $4^{\text {th }}$ ed. pp.1509-1522.

Huffnagle, G.B.; Dickson, R.P.; Lukacs, N.W. (2017). The respiratory tract microbiome and lung inflammation: a two-way street. Mucosal Immunology, 10(2), 299.

Jalal, R.; Lateef, B.; Ibrahim, V. (2017). Isolation and identification some of microbial causes for dental caries. Tikrit J. Pure Sci., 22(9), 26-29.

Janak, K. (2016). Diabetes mellitus a complex and heterogeneous disease and the role of insulin resistance as a determinant of diabetic kidney disease. Nephrology Dialysis Transplantation. 31, 206-213.

Khursheed, M.; Devrajani, B.R.; Shah, S.Z.A.; Devrajani, T.; Das, T.; Raza, S. (2011). Lipid profile of patients with diabetes mellitus (a multidisciplinary study). World Applied Sci. J., 12(9), $1382-1384$.

Kripal, K. (2017). Diabetes mellitus as a risk factor for periodontitis. EC Dental Sci., 1, S2.

Kumari, M.; Naik, S.B.; Shanbhag, N.; Pradeep, A.R. (2011). Diabetes and periodontitis: a casual link or a two way street. Archives Oral Sci. and Research. 1(4), 221-227.

Li, S.; Song, Z.; Dong, J.; Shu, R. (2017). Micro RNA-142 is unregulated by tumor necrosis factoralpha and triggers apoptosis in human gingival epithelial cells by repressing BACH2 expression. American J. Translational Research, 9(1), 175-183.

Luo, Y.; Gong, Y.; Yu, Y. (2013). Interleukin-10 gene promoter polymorphisms are associated with cyclosporin A-induced gingival overgrowth in renal transplant patients. Archives Oral Biology, 58(9), 1199-1207. 
MacDonald, K. W. (2015). The role of streptococcus salivarius as a modulator of homeostasis in the oral cavity. Electronic Thesis and Dissertation Repository, The University of Western Ontario. 2816.

Marques, C.P.; Victor, E.C.; Franco, M.M.; Fernandes, J.M.; Maor, Y. (2016). Salivary levels of inflammatory cytokines and their association to periodontal disease in systemic lupus erythematosus patients. A case-control study. Cytokine, 85, 165-170.

Mittendorfer, B. (2005). Sexual dimorphism in human lipid metabolism. The J. Nutrition, 135(4), 681-686.

Norppa, A. (2012). Association between periodontal and systemic inflammation. A Study of Proand Anti-Inflammatory Mediators. Academic dissertation to be presented with the assent of the Doctoral Training Committee of Health and Biosciences of the University of Oulu for public defense in Auditorium F202 of the Department of Pharmacology and Toxicology.

Rajole, M.; Ahire, S. (2016). Study of lipid profile in patients with diabetes mellitus. J. Cont. Med. A Dent., 4 (1), 27-30.

Satpathy, A.; Ravindra, S.; Thakur, S.; Kulkarni, S.; Porwal, A.; Panda, S. (2015). Serum interleukin-1 $\beta$ in subjects with abdominal obesity and periodontitis. Obesity Research and Clin. Practice, 9(5), 513-521.

Solis-Herrera, C.; Triplitt, C.L.; Lynch, J.L. (2014). Nephropathy in youth and young adults with type 2 diabetes. Current Diabetes Reports, 14(2), 456-473.

Stashenko, P.; Van Dyke, T.; Tully, P.; Kent, Jr.R.; Sonis, S. (2011) Inflammation and genetic risk indicators for early periodontitis in adults. J. Periodontol., 82(4), 588-596.

Tille, P.M. (2014). "Bloodstream Infections. Bailey and Scott's Diagnostic Microbiology". $13^{\text {th }}$ ed. St. Louis, MO: Mosby Elsevier, pp. 865-72.

Von Wintersdorff, C.J.; Penders, J.; van Niekerk, J.M.; Mills, N.D.; Majumder, S.; van Alphen, L. B.; Wolffs, P.F. (2016). Dissemination of antimicrobial resistance in microbial ecosystems through horizontal gene transfer. Frontiers in Microbiol., 7, 173-187.

Whary, M.T.; Baumgarth, N.; Fox, J.G.; Barthold, S.W. (2015). Biology and diseases of mice. In Laboratory Animal Medicine (Third Edition), 143-149.

Willnow, T.E.; Hammes, A.; Eaton, S. (2007). Lipoproteins and their receptors in embryonic development: more than cholesterol clearance. Development, 134(18), 3239-3249.

Yadav, K. (2016). Dental Caries: Bacterial profile of Dental caries. Lambert Academic Publishing, Omni Scriptum GmbH and Co. KG, Germany, 120.

Zhang, F.; Si, M.; Wang, H.; Mekhemar, M.K.; Dörfer, C.E.; El-Sayed, K.M. (2017). IL-1/TNF- $\alpha$ Inflammatory and anti-inflammatory synchronization affects gingival stem/progenitor cells' regenerative attributes. Stem Cells International. 2017, 9. 